\title{
A Coupled Approach to Developing Damage Prognosis Solutions
}

\author{
Charles R. Farrar ${ }^{1}$, Francois Hemez ${ }^{2}$, Gyuhae Park ${ }^{3}$ Amy N. \\ Robertson ${ }^{4}$, Hoon Sohn ${ }^{5}$, Todd O. Williams ${ }^{6}$
}

\author{
${ }^{1}$ MS T-006, Los Alamos National Laboratory, Los Alamos, NM 87545, USA farrar@lanl.gov \\ ${ }^{2}$ MS T-006, Los Alamos National Laboratory, Los Alamos, NM 87545, USA hemez@lanl.gov \\ ${ }^{3}$ MS T-006, Los Alamos National Laboratory, Los Alamos, NM 87545, USA gpark@lanl.gov \\ ${ }^{4}$ HYTEC, Inc., 4735 Walnut, Suite W-100 Boulder, CO 80301, USA, arobertson@hytecinc.com \\ ${ }^{5}$ MS T-006, Los Alamos National Laboratory, Los Alamos, NM 87545, USA sohn@lanl.gov \\ ${ }^{6}$ MS B-216, Los Alamos National Laboratory, Los Alamos, NM 87545, USA oakhill@lanl.gov
}

Keywords: Damage detection, damage prognosis, structural health monitoring, model validation, uncertainty quantification, reliability analysis, impedance method

Abstract. An approach to developing damage prognosis (DP) solution that is being developed at Los Alamos National Laboratory (LANL) is summarized in this paper. This approach integrates aadvanced sensing technology, data interrogation procedures for state awareness, novel model validation and uncertainty quantification techniques, and reliability-based decision-making algorithms in an effort to transition the concept of damage prognosis to actual practice. In parallel with this development, experimental efforts are underway to deliver a proof-of-principle technology demonstration. This demonstration will assess impact damage and predict the subsequent fatigue damage accumulation in a composite plate. Although the project focus will be DP for composite materials, most of this technology can generalize to many other applications. The unique aspects of this approach discussed herein include: 1) multi-length scale damage models analyzed on tera-scale computer platforms that discretize composites on an individual lamina level, 2) integration of advanced sensors with Los Alamos's flight-hardened data acquisition system, 3) damage detection based on a statistical pattern recognition approach, and 4) reliability-based metamodels with quantified uncertainty that can be deployed on microprocessors integrated with the sensing system for autonomous damage prognosis.

\section{Introduction}

Damage Prognosis is the estimate of a system's remaining useful life. This estimate is based on the output of predictive models that develop such estimates by coupling information from usage monitoring; structural health monitoring; past, current and anticipated future environmental and operational conditions; the original design assumptions regarding loading and operational environments, and previous component and system level testing. Also, "softer" information such as user "feel" for how the system is responding should be used to the greatest extend possible when developing DP solutions. Stated another way, DP attempts to forecast system performance by measuring the current state of the system, estimating the future loading environments for that system, and predicting through simulation and past experience the remaining useful life of the system.

An important first step in defining DP solutions is the classification of the DP problem. While it is unlikely that all or even most DP applications will fit nicely within a rigid, precise classification scheme, there are some general categories that are applicable to most problems. To understand the categories, one must first answer three general questions: 1) what is causing the damage of concern? 2) what techniques should be used to assess and quantify the damage? 3) once the damage has been 
assessed, what is the goal of the prognosis? As discussed below, the categories do not have sharp boundaries, and many applications will overlap the various categories.

For each potential failure mode, the source of the damage falls into three general categories. The first category is gradual wear, where damage accumulates slowly at the material or component level, often on the microscopic scale. Examples of this damage source include fatigue cracking and corrosion. The second category is predictable discrete events. While the damage typically still originates on the microscopic scale, it accumulates at faster rates during sudden events that can be characterized a priori. Examples include aircraft landings and explosions in confinement vessels. Unpredictable discrete events make up the third category in which unknown and usually severe insults are inflicted upon the system at essentially unpredictable times. Examples include foreignobject-induced fan blade-off in turbine engines, earthquake-induced damage in civilian infrastructure, or battle damage military hardware.

After identifying the type(s) and source(s) of damage, it is then important to determine which techniques should be used in the damage assessment. The first question that arises concerns whether the assessment should be done on-line, in near-real-time, or off-line at discrete intervals, as this consideration will strongly influence the data acquisition and data processing requirements, as well as set limits on the computational requirements of potential assessment and prognosis techniques. While it is obvious that measurements should be taken on-line, the type of damage will influence whether the assessment needs to be done on-line. That is, for unpredictable discrete events, the assessment must be done on-line to be of any use, thus limiting the choice of the assessment techniques. However, for gradual wear, there are cases where the assessment need not be performed in near real time, and virtually any assessment technique may be used.

Assessment techniques can generally be classified as either physics-based or data-based, though practically speaking, a combination of the two will usually be employed. The physic-based assessments are especially useful for predicting system response to new loading conditions and/or new system configurations (damage states). However, physics-based assessment techniques are typically computationally intensive.

Data-based assessment techniques, on the other hand, rely on previous measurements from the system to assess the current damage state, typically by means of some sort of pattern recognition method, such as neural networks. However, although data-based assessment techniques may be able to indicate a change in the presence of new loading conditions or system configurations, they will perform poorly when trying to classify the nature of the change. Thus, it is not uncommon to use the results from a physics-based model to "train" a data-based assessment technique to recognize damage cases for which no experimental data exists. Typically the balance between physics-based models and data-based techniques will depend on the amount of relevant data available and the level of confidence in the physics-based models.

Once the current damage state has been assessed, the prognosis problem can begin to be addressed by determining the goal for the prognosis. Perhaps the most obvious and desirable type of prognosis estimates how much time remains until maintenance is required, the system fails, or the system is no longer usable. While this estimate is of high value in systems where damage accumulates gradually and at predictable rates, it is of less value in more extreme conditions such as fighter aircraft in combat, where the users of the system (the pilot and mission commander) really want to know the probability of completing the current mission, given the current assessment of the damage state. Because predictive models typically have more uncertainty associated with them when the structure responds in a nonlinear manner as will often be the case when damage accumulates, an alternate goal might be to estimate how long the system can continue to safely perform in its anticipated environments before one no longer has confidence in the predictive capabilities of the models that are being used to perform the prognosis.

Having defined the DP problem, we propose to develop DP solution by integrating advanced state awareness sensing hardware, numerical simulations of unprecedented fidelity that model the damage evolution process, and data interrogation procedures that couple the measurements and 
simulations to estimate remaining system life with quantified uncertainty. This solution procedure will make extensive use of reliability-based decision-making algorithms to transition the concept of DP to actual practice. This discussion begins by first providing an outline of the entire DP process. Next, the demonstration problem that is providing the framework around which to develop the DP process is presented. This paper then summarizes the technologies that are being developed and integrated to address the DP problem.

\section{The Damage Prognosis Process}

Figure 1 summarizes the DP process and tries to identify the portions of this process that are data based and the portions that rely on a physics-based model. The process begins by collecting as much initial system information as possible. This information is used to develop initial physicsbased numerical models of the system as well as to define the sensing system that will be used for state awareness assessments and whatever additional sensors are need to monitor operational and environmental conditions. The physics-based models will also be used to define the necessary sensing system properties (e.g. location, bandwidth, sensitivity). As data become available from these sensing systems, they will be used to validate and update the physics-based models. These data along with output from the physics-base models will also be used to assess the current state of the structure (existence, location, type and extent of damage). Data from the operational and

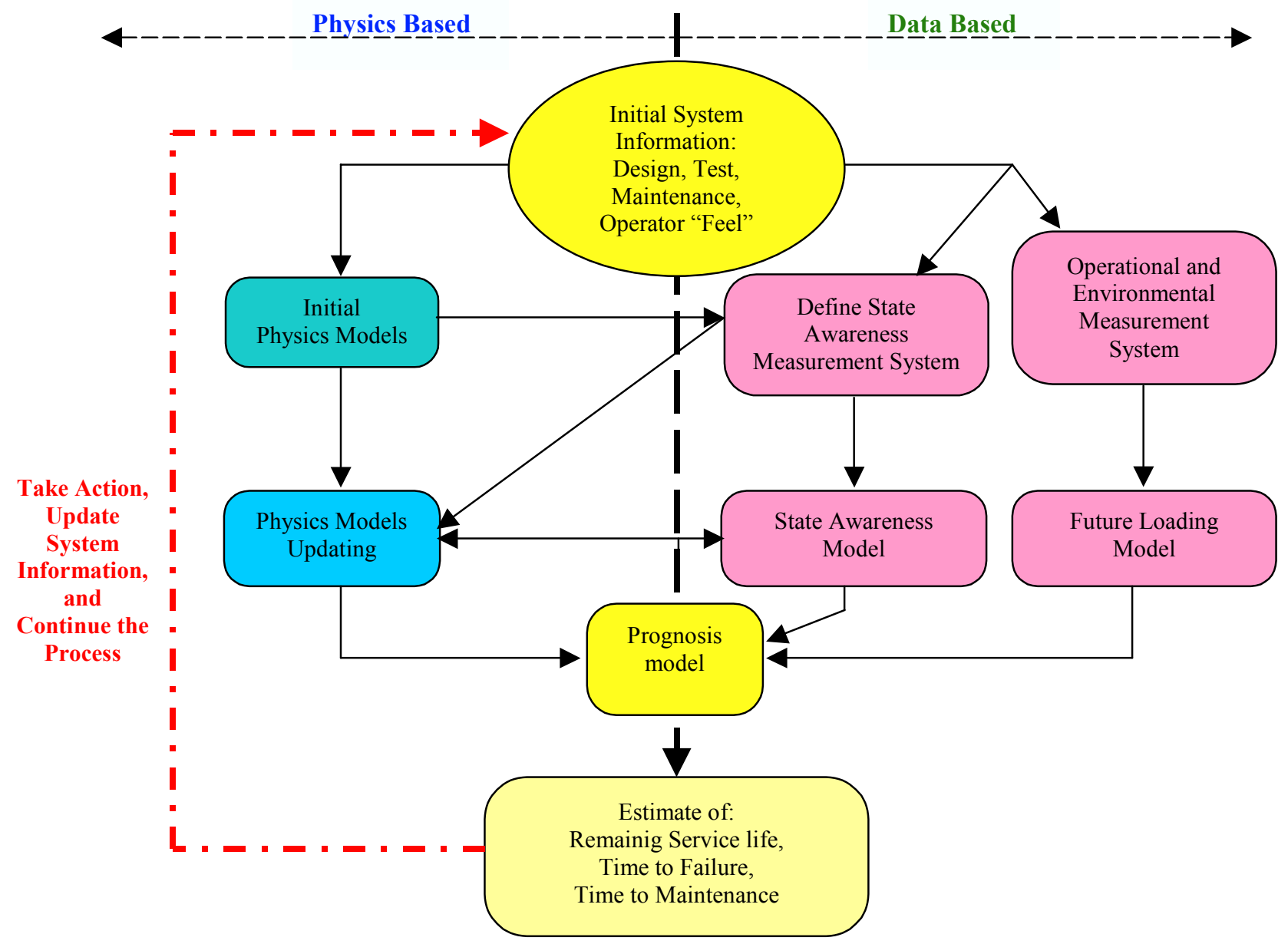

Figure 1: A general damage prognosis solution procedure showing the interaction of data-based and physics-based assessment and predictions with the other issues. 
environmental sensors will be used to develop data-based models that predict the future system loading. The output of the future loading model, state awareness model, and the updated physicsbase model will all be input into a reliability based predictive tool that estimates the remaining system life. Note that the definition of "remaining life" can take on a variety of meanings depending on the specific application. From this figure it is clear that various models will have to be employed in the prognosis process. Also, the data-based and physics-based portions of the process are not independent. As is indicated, the solution process will be iterative, relying on experience gained from past predictions to improve future predictions

\section{Demonstration Problem}

To simulate damage to an aircraft wing or fuselage in a laboratory setting, we are conducing controlled projectile impact experiments on a graphite/epoxy fiber reinforced composite plate using the gas gun shown in Fig. 2. A 185-gram, 5-cm-long, 2.5-cm-diameter, A2 steel projectile with a spherical nose was used. The composite plates are instrumented with both strain gages and accelerometers. Also, the piezoelectric active sensing system discussed below is being used to interrogate the plate with high frequency pulses whose transmission path quantifies damage location and extent. The recorded data is being used to detect and quantify damage to the composite plate. Modal impact hammer tests are conducted prior to and following projectile impact. Impulse response waveforms resulting form the hammer impacts are used to train the impact location metamodel and to quantify structural vibration frequencies, damping values, and mode shapes. The damaged composite plate is then subjected to steady-state cyclic loading on a shake table a shown in Fig. 3. Successive tests are conducted with the shake table input set to a 10 -g amplitude excitation at the first resonant frequency of the plate with the intent of propagating damage from the projectile impact site to a larger region of the plate. The damaged composite plates are subjected to ultra-sonic inspection to quantify the location and size of the damage. The first test plate has been subjected to all tests described above in an effort to check out the test procedure and instrumentation. Currently, preparations are underway to test the first plate that is instrumented with the active sensing system

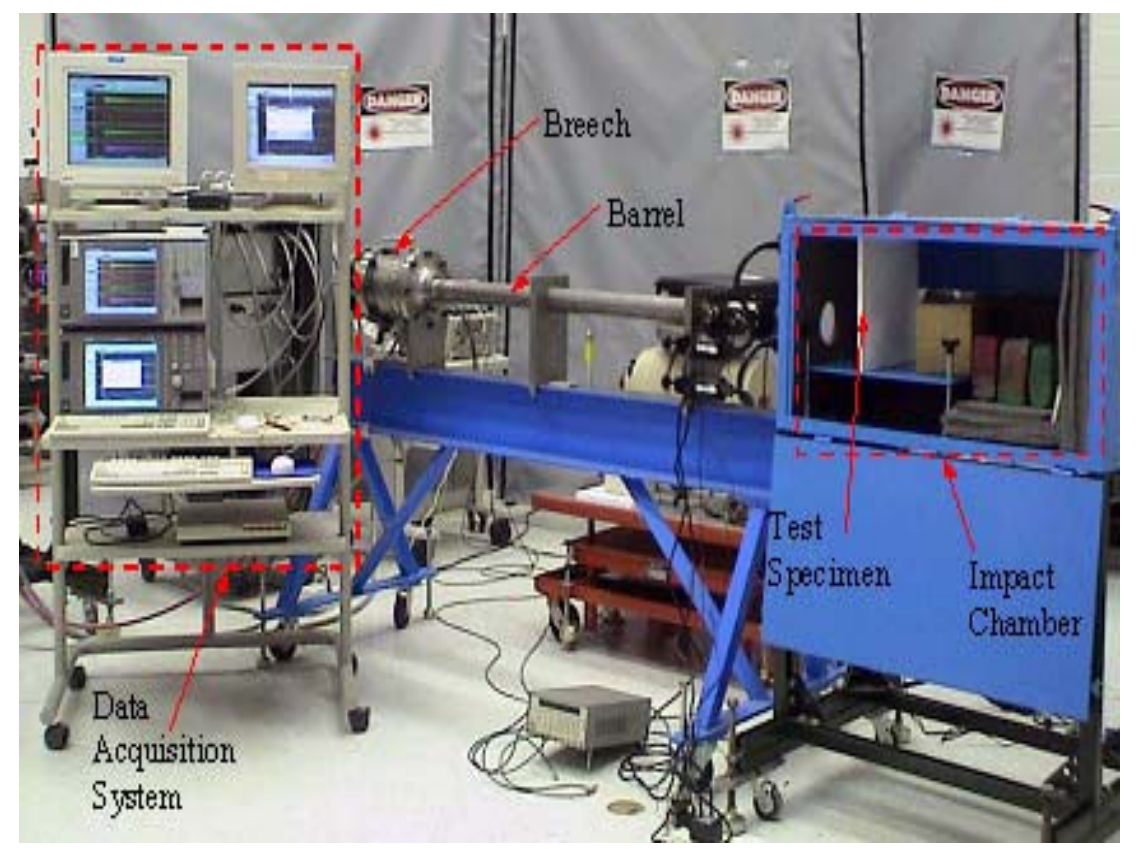

Fig. 2 Gas gun facility used to impact composite plate 


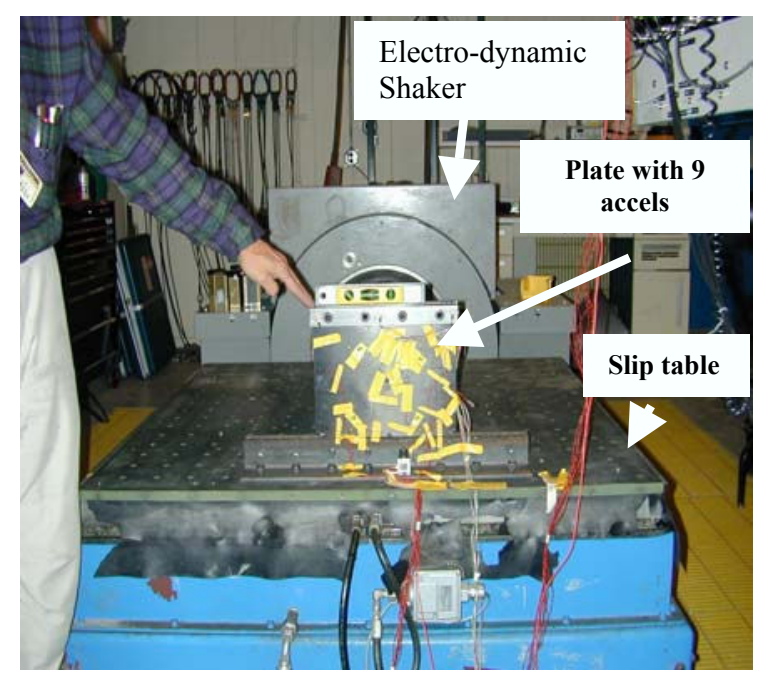

Fig. 3 Composite plate mounted on shake table for fatigue testing

\section{Multi-length-scale, physics-based models for damage initiation and propagation}

The purpose of this component of the program is to develop an appropriate physics-based theoretical framework that is being used to carry out deterministic analyses of composite materials in the presence of impact and fatigue loading. Additionally, this work can be used to assist in the development of the experimental tests, define sensing system properties as well as aid in the development of the reduced order prognosis models suitable for deployment in microprocessors deployed onboard the structure.

A composite structure is composed of lamina stacked with specified orientations relative to the global coordinate system. The lamina are in turn composed of continuous fibers embedded in a polymer matrix. Thus, there are three distinct length-scales associated with the composite plate response (the total plate thickness, the lamina thicknesses, and the fiber diameter). Within the context of this type of structure there are a number of possible damage modes and other history dependent phenomena that must be considered. At the smallest length scale (the fiber level) it is necessary to model viscoelasticity in the matrix, debonding between the fiber and the matrix, fiber failure, and matrix failure. All of these processes evolve in a highly nonlinear fashion.

To correctly predict the evolution of these responses, it is necessary to have accurate assessments for the local deformation and stress states in the lamina. The history-dependent phenomena at the smallest length scale tend to drive the initiation and evolution of the nonlinear (inelastic and damage) behavior at the larger length scales. In particular, the damage mode at the lamina level is intraply cracking (along the fiber direction). Such intraply cracking in turn typically initiates the evolution of delaminations at the lamina interfaces. The overriding factor driving the continued evolution of delaminations is the local transverse stress state in the laminated plate. While the above discussion has proceeded from the lower length scale to the highest it is important to note that the behaviors at all length scales are mutually interactive. The above considerations imply that an appropriate physics-based modeling framework for this project must accurately predict the local behavior in the lamina within the composite structure as well as incorporate fracture mechanics considerations at all three length scales.

In order to correctly address the modeling issues associated with the composite material response, a novel multi-length scale finite element (MSFE) approach developed at LANL is being used [1]. The MSFE theory is based on a new generalized, nonlinear, multi-length scale plate theory shown schematically in Fig. 4. 


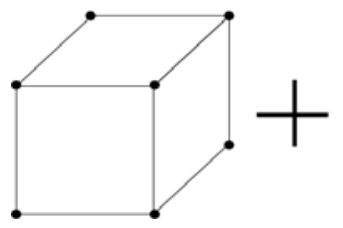

Global coordinates

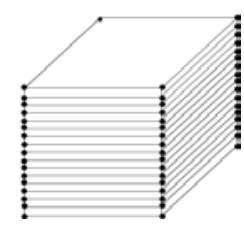

Local Coordinates

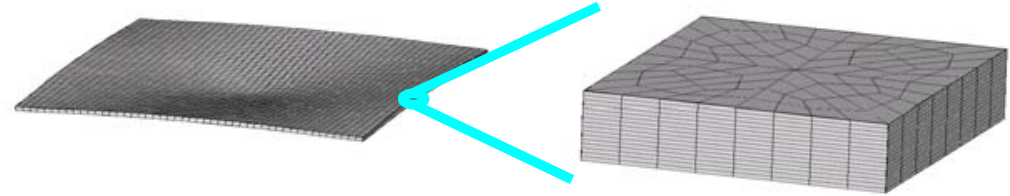

Individual lamina modeled in local coordinate system

Fig. 4 Multi-length scale composite plate modeling approach where each lamina is discretely modeled in a local coordinate system using homogenization

This plate theory has been formulated in terms of arbitrary global and local displacement expansions. The local field aspect of the theory is necessary in order to correctly model delamination behavior. The theory accounts for the initiation and evolution of delaminations between lamina through the use of cohesive zone models (CZMs) [2]. This plate theory has been shown to accurately predict the local elastic fields within a plate as well as the global plate buckling loads in the presence of both perfect bonding between lamina as well as in the presence of delaminations [3]. While the basic plate theory is capable of utilizing any CZM, the MSFE model utilizes either a simple bilinear model or a more advanced CZM that incorporates Coulombic frictional effects. Introducing the concept of super-elements into the MSFE formulation has resulted in a hierarchical analysis for the plate behavior that exhibits enhanced computational efficiency without adversely affecting the accuracy of the predictions for the local fields.

Currently the material modeling has been carried out using homogenization techniques and the assumption of linear elastic behavior for the fibers and matrix [4]. Homogenization is the process of combining matrix and fiber material properties into a single material that is representative of the individual lamina. Next, nonlinear homogenization models, currently available at LANL, are being implemented into the MSFE framework. These types of theories correctly predict the localizations with the material microstructure necessary to accurately determine both the history-dependent deformations and fracture behavior at the fiber level as well as the bulk response of the lamina.

In this project advanced physics-based modeling are being developed in the following manner. We will develop a strong form solution to replace the current variationally derived theory. This new theory will utilize the same basic global and local expansion for the displacement fields, but will satisfy strong form of the governing equations of continuum mechanics. The advantage of the new approach is that it will satisfy the traction continuity conditions between lamina in a strong sense. Satisfying the strong form of the traction continuity conditions will increase the ability of the plate theory to provide high fidelity predictions of the delamination behavior.

This modeling approach is being deployed on LANL's tera-scale super computers (ASCI-Q, a 8000 processor, 20 tera-ops machine currently ranked as the second most power computer in the world. Such a computing platform provides the opportunity to model damage evolution in composite materials with a level of fidelity never before possible. This material modeling approach coupled with the tera-scale computing resources can provide a solution to the problem of predicting system level response in composite structures on widely varying length and time scales. When coupled with uncertainty quantification methods described below, this modeling approach will be used to assess the influence of residual stresses and initial flaws on the DP predictions. Finally, these high-fidelity models are being used to define the required sensing system capabilities so this system can be designed to provide the requisite sensitivity before it is actually deployed on the structure. 


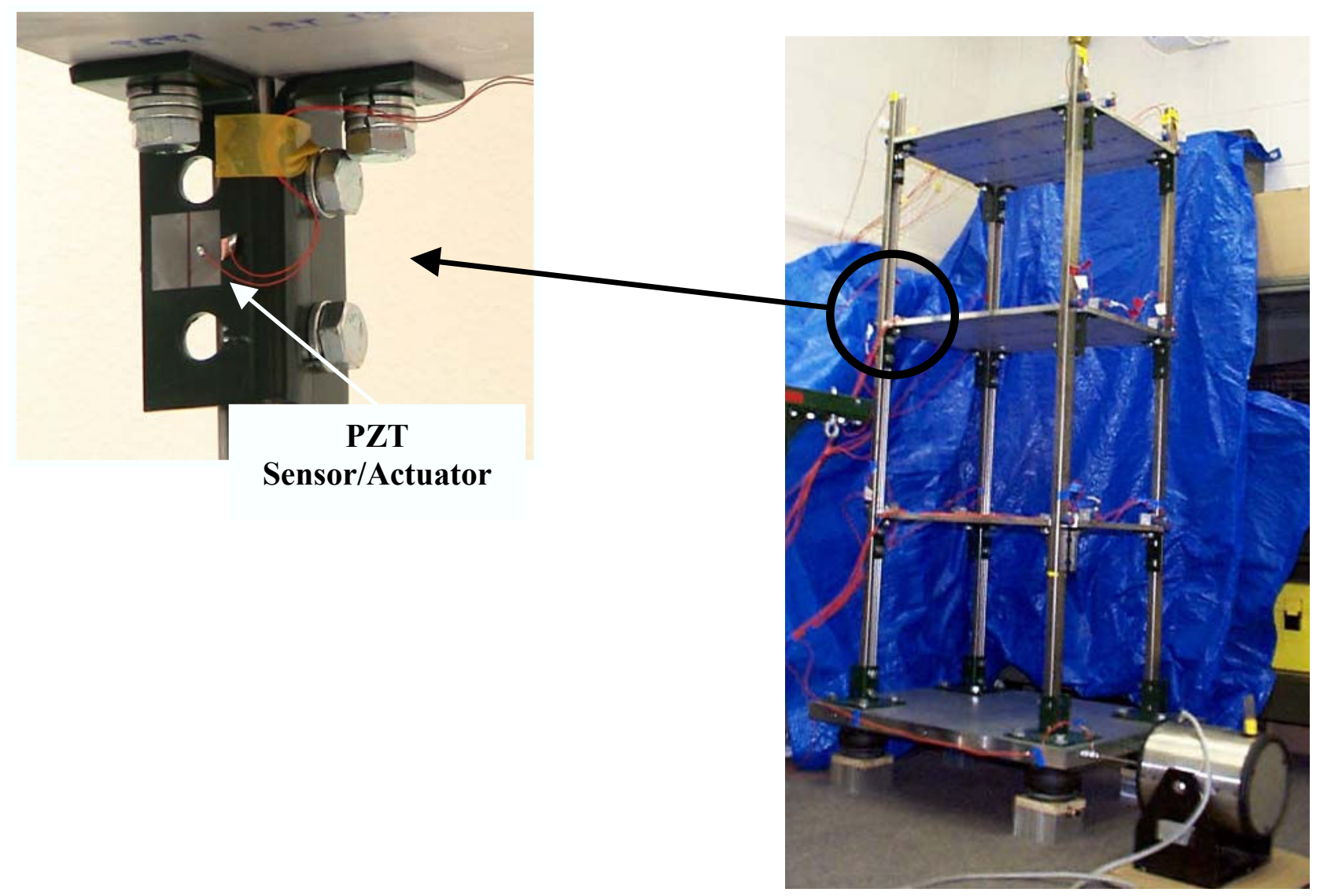

Fig. 5 PZT sensor/actuator being used to monitor a bolted connection.

\section{State Awareness Sensing}

The proposed prognosis sensing strategy is broken into three major parts: 1) active and local sensing for monitoring of incipient and propagating damage, 2) passive global sensing to assess systemlevel response and loading conditions, and 3) development of a sensing system design methodology that aims to maximize the observability of a given failure mode and degradation mechanism. Initially, the hardware aspect of this work will focus on using recently developed advanced sensing technology (active piezoelectric (PZT) sensors) as well as conventional off-the-shelf sensors (standard piezoelectric accelerometers).

Local Active Sensing: In this project, PZT is being used as both sensors and actuators to form an "active" local sensing system. Because PZT produces an electrical charge when deformed, PZT patches can be used as dynamic strain gauges. Conversely, the same PZT patches can also be used as actuators because a mechanical strain is produced when an electrical field is applied to the patch. The use of a known and repeatable input makes the subsequent signal processing for damage detection and prognosis much easier. These sensor/actuators are inexpensive (approx. \$5/PZT patch), generally require low power, and are relatively non-intrusive as shown in Fig. 5.

Examples of documented successes in the areas of active local sensing using PZT are the impedance-based damage detection method [5] and the Lamb wave propagation method [6]. The impedance method monitors the variations in mechanical impedance resulting from damage, which is coupled with the electrical impedance of the PZT sensor/actuator. For the Lamb wave propagation method, one PZT is activated as an actuator to launch elastic waves through the structure, and responses are measured by an array of the other PZT patches acting as sensors. The structure can be systematically surveyed by sequentially using each of the PZT patches as an actuator and the remaining PZT patches as sensors. The technique looks for the possibility of damage by tracking changes in transmission velocity and wave attenuation/reflections. Both 


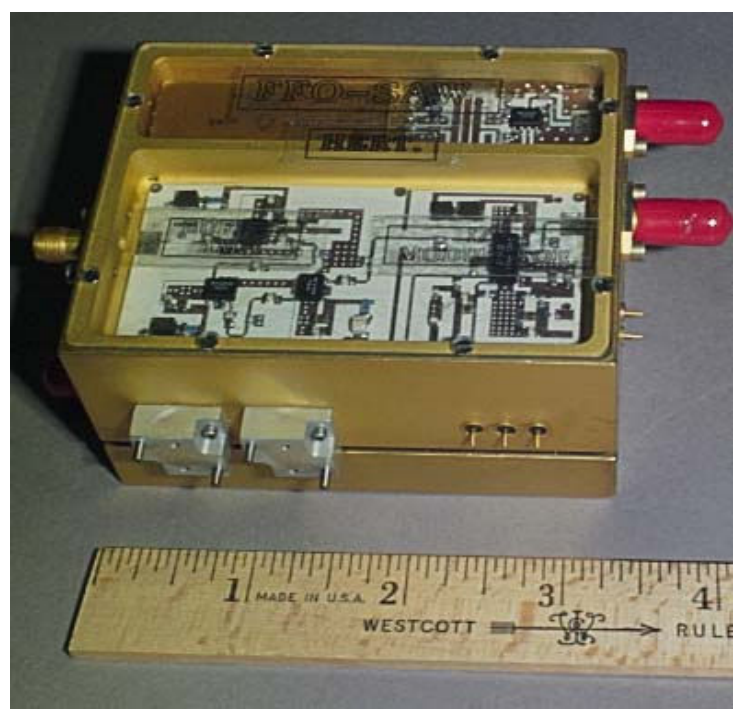

Fig. 6 High explosives radio telemetry system that will be adapted for sensor system processing and telemetry.

methods operate in the high frequency range (typically above $30 \mathrm{kHz}$ ) where there are measurable changes in structural responses for even incipient damage associated with crack formation, debonding, delamination, and loose connections.

Global Sensing: Once structural damage has initiated and is detected, numerical models will be used to capture the influence of damage on the system level performance and predict future performance. This procedure necessitates the measurement of system level response. For the demonstration problem commercially available piezoelectric accelerometers will be used to assess the global response of the plate to various loading conditions. These sensors will also be used to quantify the coupling between the first bending and torsion modes, which will simulate the influence of the local damage on the flutter characteristics of a wing.

We believe that for field applications on actual aircraft, the accelerometers will be replaced with fiber optic strain sensors based on Bragg grating technology [7]. Multiple Bragg gratings can be placed in a single long fiber to obtain numerous discrete strain readings. These readings can be obtained with greater accuracy than electrical resistance strain gages, the readings are immune to electromagnetic/RF interference, and the sensors are not a spark source, which is a key issue if monitoring is to be done near combustible materials. Also, these sensors are non-intrusive, extremely lightweight and have proven to be very rugged [8].

Optimal Sensing System Design Strategy: Such a strategy demands a significantly coupled analytical and experimental approach to the sensor system deployment. According to control theory, the observability of a system relies critically on the location of the sensors and the desired feature to be extracted. We will examine the issues associated with the integration of observability indices for local damage and global behaviors of a system into the optimal sensing design, which has not been addressed in current damage identification practice. Such methods will undoubtedly incorporate genetic algorithms [9] or neural networks with the ability to model the system in detail. Additional sensing requirements will also be considered based on how changing operational and environmental conditions affect the damage detection process.

The High-Explosives Radio Telemetry (HERT) system, Fig. 6, developed at LANL for weapons flight test monitoring will be the basis of this integrated DP sensing and processing system. The HERT system currently can measure, record, process and transmit 64 fiber optic sensor channels. A field programmable gate array is used for local data processing. Our motive for selecting HERT as the starting point for a sensing system is the fact that the HERT system has been hardened for 
intercontinental ballistic missile flight and, hence, will be rugged enough for field deployment on aircraft and ship structures. Finally, in conjunction with adapting the HERT system to DP tasks, we will integrate the PZT patches previously described for local active sensing into the HERT system. In addition, these same PZT patches will be used in a passive mode for global system monitoring. Software embedded in the HERT system processor will be used to control the system allowing it to switch between passive global sensing mode and the active local sensing mode.

\section{Data Interrogation for system state quantification}

The main issue of data interrogation is how to utilize a combination of simulated and experimental data to support final DP decision-making. Data interrogation for this project will encompass many aspects of data analysis, signal processing, machine learning techniques, data management, and feature extraction. The interaction of data interrogation with the other tasks includes the followings: First, the model updating and refinement requires experimental test data to adjust and validate the numerical model. In this process, the data interrogation condenses the output of massive numerical data and extracts features from physical measurements for comparison with features extracted from the numerical data. The data interrogation component of the damage diagnosis process defines damage-sensitive features and formulates statistical procedures to determine the existence, location, and extent of damage. Damage prognosis results from the coupling of the current system state determined from the damage diagnosis process, estimated future loading information, and the predictive capability of the previously refined model. The future loading can be forecast using various data-driven prediction modeling techniques. Then, a decision analysis similar to the one in the damage diagnosis step will be designed to synthesize all this information in an effort to make the DP. The data interrogation methods needed for this process are data validation, feature extraction, data normalization, characterization of feature distributions, statistical inference for decision-making and prediction modeling for future loading estimates.

Data Validation. The first step in data interrogation is to inspect data obtained from sensing devices or numerical simulations. The data validation step should assure that the recorded data possess information relevant to subsequent analysis. For instance, measured acceleration data can have anomalies because of electromagnetic interference, missing values because of disrupted RF transmission, or DC offset caused by drift. Data validation techniques can also be conducted to monitor the functionality of sensors themselves or the bonding condition of the instrumentations to the host structure. For instance, some standard features such as autospectra, frequency response functions, modes, response probability density will be available in near-real-time to analysts and statisticians, allowing data validation during data acquisition, not after the fact when it is too late to modify test measurement parameters. Statistical inference techniques such as outlier analysis [10] and novelty detection will be used in this data validation process.

Feature Extraction. Because new sensing technologies are enabling structures to be instrumented with large sensor arrays and new computation capabilities produce substantial amounts of numerical data, it is essential to compress these data for subsequent analyses, keeping useful information relevant to later analysis. Feature extraction refers to a procedure to identify the salient features of data to facilitate its use in subsequent analyses, in the current case, damage diagnosis and prognosis [11]. Almost all feature extraction procedures inherently perform some form of data compression. Data compression into feature vectors of small dimension is necessary if accurate estimates of the feature's statistical distribution are to be obtained. The aspects of a specific problem must be considered in the feature extraction process. Also, nonlinear features will be necessary for damage detection because the propagation of damage often produces nonlinear system responses. Data interrogation techniques will assist analysts with various tools such as linear/nonlinear principle component analysis [12], Fisher's discriminant [13], and Independent Component Analysis [14]. 
For this project, damage-sensitive features will be extracted from wave propagation and impedance data obtained from piezoelectric sensor/actuators mounted on the plate. The main goal of this signal processing is to locate and quantify the area of hidden delamination based on changes in electrical impedance or dispersion and attenuation characteristics of the signal transmitted from one actuator to adjacent sensors. Preliminary studies indicate that a delamination covering an area of less than $1 \%$ of the composite plate can be identified using the dispersion and attenuation characteristics of the Lamb waves as features. Wavelet based signal processing techniques will be developed to improve the resolution of this damage detection.

In addition, a Holder exponent technique will be used to detect the presence of signal discontinuities caused by damage such as the rattling of loose components, and to identify when they occurred. The Holder exponent provides a measure of a signal's regularity, which is defined as the number of continuous derivatives that the signal possesses. The usefulness of this method has been shown both for data that contains an artificially introduced discontinuity and to an actual engineering system that has a rattle [15].

Data Normalization. Damage diagnosis is based on the examination of a system's dynamic response to determine if the system significantly deviates from an initial baseline condition. In reality, the system is often subject to changing environmental and operation conditions that affect its dynamic characteristics. Such variations include changes in loading, boundary conditions, temperature, and moisture. Most damage diagnosis techniques, however, generally neglect the effects of these changing ambient conditions. For the development of robust monitoring systems, these natural variations of the system responses will be explicitly taken into account in order to minimize false positive indications of true system changes. Auto-Associative Neural Networks [16] and a combination of Auto-Regressive (AR) and Auto-Regressive with Exogenous Inputs (ARX) models [17] have been developed at LANL to address this data normalization issue. Furthermore, Time-dependent Auto-Regressive Moving-Average (TARMA) models [18], and Evolutionary Spectral Analysis [19] are being investigated. These time-varying time series and spectral analyses have found wide applications in modeling of nonstationary processes and various naturally occurring phenomena.

Characterization of Feature Distributions. Because of inherent uncertainties involved in data measurements and physical model development, the decision-making procedures for damage diagnosis and prognosis should be based on statistical modeling of feature spaces. There are varieties of tools for decision analysis. To name a few, Hypothesis Testing [20] Outlier \& Novelty Detection [10, 21], Sequential Probability Ratio Tests [22], Statistical Process Control [23] Group Clustering, and Bayes Decision Theory [24]. These techniques require parametric or nonparametric characterization of feature distributions or the estimation of statistical properties of the assumed distribution. For example, outlier analysis demands the estimation of a probability density function for the features corresponding to an undamaged state of a structure. Furthermore, inherent uncertainties should be addressed in this decision-making process. For example, how does uncertainty in damage diagnosis propagate through to uncertainty in DP? A review of uncertainties in dynamic analysis can be found in Langley [25] and uncertainty quantification is further discusses in Section 1.B.3.4.

Statistical Inference for Damage Diagnosis. Statistical inference is concerned with the implementation of algorithms that analyze the distribution of extracted features in an effort to make decisions on damage diagnosis and prognosis. The algorithms used in statistical model development fall into the three general categories: (1) Group Classification, (2) Regression Analysis, and (3) Outlier Detection. The appropriate algorithm to use will depend on the ability to perform supervised or unsupervised learning. Here, supervised learning refers to the case where examples of data from damaged and undamaged structures are available. Unsupervised learning refers to the case where data are only available from the undamaged structure ${ }^{11}$. The success of decision-making can be assessed by (1) overall misclassification rate (false positive/negative 


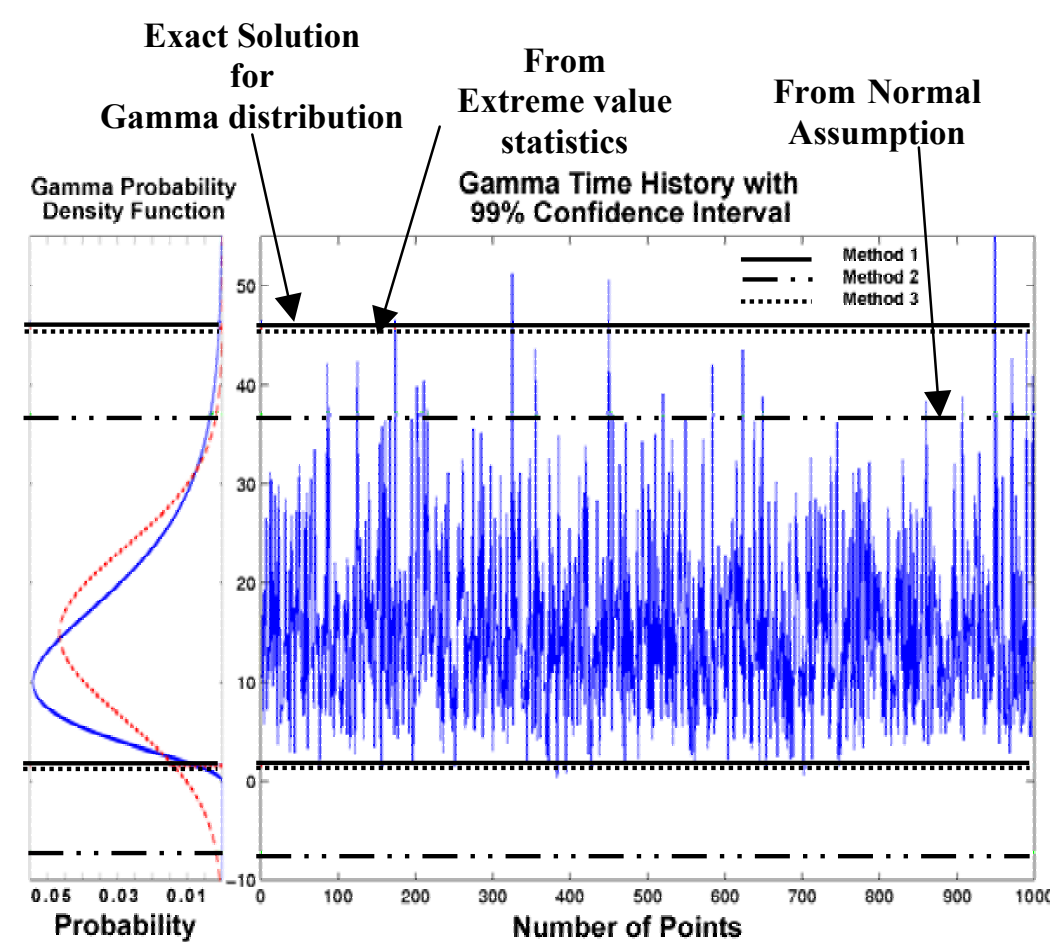

Fig. 7 The increasing false-positive and false-negative outlier detection if confidence limits are based on a normality assumption when underlying feature has a Gamma distribution. Extreme value statistics based on Gumbel distribution accurately represent the true thresholds.

indications of damage or system failure), (2) Receiver Operating Characteristic [26] curves (ROC), and (3), confidence intervals on prediction.

One of the main issues in this decision-making procedure is to establish decision threshold values. In particular, Extreme Value Statistics [27] will be employed for the establishment of decision boundaries to minimize false positive and negative indications of damage. Statistical inference is often based on the assumption that the underlying distribution of data is Gaussian. However, the assumption of normality imposes potentially misleading behavior on the extreme values of the data, namely, those points in the tails of the distribution as is illustrated in Fig. 7. As the problem of damage identification specifically focuses attention on these tails, the assumption of normality is likely to lead any analyses astray. An alternative approach based on Extreme Value Statistics is developed to specifically model behavior in the tails of the distribution of interest. Furthermore, the development of statistical models should vary depending on the targeted damage scenarios. For example, it will be necessary to track trends in feature spaces for the detection of slowly accumulating deterioration over long time periods as opposed to sudden anomalies in features resulting from a discrete event. Uncertainties and propagation of uncertainties will be taken into account in the establishment of decision criteria.

Prediction modeling for future loading estimates. A successful DP requires the measurements of the current system state, and the prediction of the system deterioration when subjected to future loading. Based on the analysis of previous loading histories, future loading will be forecast using various data-driven prediction modeling techniques. For example, metamodeling such as state space representation [28] and multivariate ARMA models [29] will be employed to track previous loading and predict future loading for this purpose. Then, decision analysis (discussed below) will be designed to synthesize all this information. 


\section{Metamodels for Damage Prognosis}

The high-fidelity, multi-scale physics models can provide unprecedented predictive capability for damage initiation and evolution, but these models are not suitable for on-board, near-real-time DP assessments. To address this issue, we are developing reduced order models referred to as metamodels that are based on outputs from the large-scale physics-based models. Metamodels capture the relationship between inputs and outputs without providing a detailed description of the physics and geometry. Examples include polynomials, exponential decays, and neural networks. Metamodels can run on microprocessors and therefore can be directly integrated with the sensing system. The process of developing the metamodels requires the ability to validate the physics-based models and the ability to quantify and propagate uncertainty through these models. Validation refers to the adequacy of a model to describe a particular physical phenomenon, and requires a comparison of the predictions with data collected during a series of physical experiments.

Design of Experiments. Metamodels must be "trained," to identify their unknown parameters. Their quality must also be evaluated independently from the training step. Because analyzing a detailed finite element model at every combination of input variables is computationally prohibitive, training is generally based on a subset of carefully selected runs. Design of Experiments (DoE) [30] techniques will be used to explore large design spaces and to select a judicious subset of finite element analyses. Metamodeling and effect screening can be performed using DoE because identifying the effects and interactions that capture a particular input-output relationship controls the functional form of the metamodel.

Uncertainty Quantification and Propagation. The goal of uncertainty quantification is to characterize the variability of prognosis predictions caused by uncertainty in the input variables. The conventional approach is to represent the uncertainty in terms of a probability density function, which provides an appropriate representation of material uncertainty and load variability. Other theories to represent uncertainty such as interval arithmetic, fuzzy logic [31], Dempster-Shafer theory of plausibility and belief [32], and information-gap [33] will be considered for representing sources of uncertainties that are difficult to represent in a conventional probabilistic manner as discussed below.

Two types of analyses are used for uncertainty quantification. The first one is the propagation of uncertainty and the second one is effect analysis. Uncertainty propagation assesses the effect of random inputs (input variables of a model or controllable parameters of a physical experiment) on the outputs. If one assumes the probability density functions for the input parameters, then sampling techniques such as Monte Carlo and Latin Hypercube sampling can be used to propagate uncertainty [34]. The outcome of the forward propagation of uncertainty is an estimation of the Joint Probability Density Function (JPDF) of the outputs, from which statistics such as the mean and standard deviation can be calculated.

To perform model calibration and screening experiments, it is also necessary to deploy techniques that can propagate uncertainty in the inverse direction, that is, from output features and damage indicators back to the input variables. To do so, we have investigated the use of Markov Chain Monte Carlo sampling and one of its variants, the Gibbs sampler [35]. The main advantage of these techniques is that they can sample an unknown JPDF, therefore, outperforming optimizationbased techniques that must assume the form of the probability law. Our current assessment is that the aforementioned sampling algorithms work well provided that the computational burden associated with multiple model evaluations can be mitigated by the use of fast-running metamodels.

The second type of uncertainty analysis involves screening experiments. Effect screening answers questions such as: "Which input parameters or combinations of these parameters are responsible for explaining output variability?" Effect screening is typically performed to reduce the number of parameters by determining which ones most influence the output features over an operational domain. Effect screening is performed through the conventional analysis-of-variance [30] and the more recently developed Bayesian screening techniques [36]. Bayesian screening 
offers a procedure for updating a priori probability information that results from the analyst's experience. Our approach, proven successful when applied to various engineering applications [37,38], is to search for consistent results when screening is performed using various techniques.

Assessment of Predictive Accuracy. Predictions will be compared to measurements to calibrate the models and assess their predictive accuracy over the entire range of potential input conditions. Of particular interest will be the extrapolation of predictive accuracy to regimes that have not been tested previously. Note this assessment can be used to define a subsequent test that provides the most information regarding the model's prediction accuracy. Besides test-analysis correlation and parametric calibration, the techniques previously mentioned (such as uncertainty quantification, effect analysis, and metamodeling) are brought to bear. For example, statistics of predictive accuracy are obtained by exercising the sources of uncertainty and assessing how such variability affects the output features for damage indicators we are trying to predict. Note that uncertainty in the context of model validation should not be restricted to parametric variability, as it is often the case. It should also include uncertainty regarding the modeling assumptions ("Is this interaction quadratic or cubic?", "Should this coupling between matrix breakage and fiber splitting be considered?", etc.). Several applications have demonstrated the benefits of validation [39]. These same validation tools will be refined and applied to this DP study to estimate when predictions can no longer be trusted.

The unknown unknowns. Finally, we are developing procedures for decision-making in the face of non-probabilistic uncertainty. One source of uncertainty that affects the experimental and simulation results is the initial condition of the structure in terms of residual stress state and distribution of initial flaws. It may not be possible to account for such complex initial conditions in the M\&S. Furthermore deriving probabilistic information about a distribution of residual stresses and initial flaws might require a prohibitive number of physical specimens and subsequent destructive evaluations. One could easily think of many other effects that even well controlled experiments or high-fidelity models will not be able to account for. In such cases, the question is not so much how to represent the uncertainty, but rather what the effect of the "unknown unknowns" is on the decision. This potential roadblock is being addressed by applying the information-gap theory to study the robustness of DP decisions with respect to non-probabilistic sources of uncertainty ${ }^{33}$.

\section{Reliability Analysis for Damage Prognosis Decision Making}

In this project, the DP forecasts will be performed in the context of reliability analysis. In reliability analysis, the failure state of a system is represented by a function of the response known as the limit state. Then, the probability of failure is the integral of the JPDF over the unsafe region bounded by the limit state as shown in Fig. 8. For the demonstration problem, a probabilistic reliability analysis will answer the question of how many more fatigue cycles can be applied before the damage reaches a critical size. If failure is defined, for example, in terms of a wing flutter condition, then reliability analysis consists of estimating the probability of reaching this limit state given uncertainties about the predictive model, current health of the system and expected loading. Decision-making relies on the estimation of reliability, as well as a quantification of its confidence, to decide which course of action should be taken.

This analysis begins with identifying the failure modes (such as delamination) and the random variables that contribute to these failure modes (such as projectile velocity, ply orientation angles, homogenized elasticity parameters, material density). To calculate the probability of failure, the JPDF must be integrated across all random variables for the failure region. Because closed-form representations of the failure region are generally not available, integration must be approximated by applying Monte Carlo sampling or approximate expansion methods [40] to the previously identified metamodels. We will apply reliability analysis to estimate the remaining useful life of the systems investigated under uncertainty. 


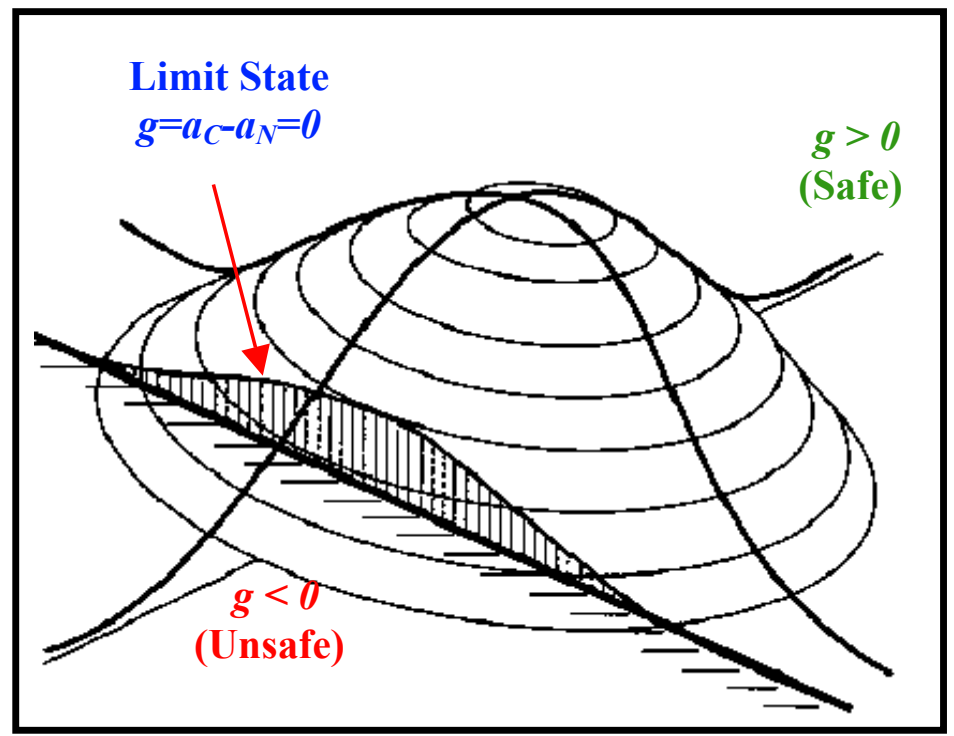

Fig. 8 Joint Probability Density Function and boundary that defines the safe and unsafe regions

\section{Summary}

The Damage Prognosis Initiative at Los Alamos National Laboratory will develop and integrate new sensing hardware, new data interrogation software, and new predictive modeling software components that will be demonstrated and further refined through component and system level proof-of-principle experiments. The program has just begun and this paper was intended to provide an overview of the issues that must be addressed and technical approaches being contemplated to realize solutions to this problem. The near term goal is to first perform the entire process on simple test specimens such as the composite plate described above and quantify the uncertainty in the prognosis introduced during each step of the process. With this information research efforts can then be concentrated on the portions of the problem contributing most to the uncertainty in the predictions of remaining system life. It is hoped that the damage prognosis solutions developed under this initiative can be used to confirm system integrity to normal and extreme loading environments, to estimate the probability of mission completion and personnel survivability, to determine optimal times for preventive maintenance, and to develop appropriate design or operation modifications that prevent observed damage propagation.

\section{Acknowledgement}

Funding for the Los Alamos Damage Prognosis Initiative is being provided by the Department of Energy through Laboratory Directed Research Development. In addition to the authors, the Damage Prognosis research team includes Los Alamos staff members Matt Bement, Irene Beyerlein, Norm Hunter, Cheng Liu, Brett Nadler, and Jeni Wait. Los Alamos graduate research assistants Tim Fasel, Jan Goethals and Trevor Tippetts, and. Professor Dan Inman and graduate student David Allen at Virginia Tech.

\section{References}

1. Williams, T.O., "A Generalized Multilength Scale Nonlinear Composite Plate Theory with Delamination,” Int. J. Solid Struc., 36, (1999), pp. 3015-3050. 
2. Corigliano, A. 'Formulation, Identification, and Use of Interface Models in the Numerical Analysis of Composite Delamination", Int. Journal of Solids and Structures, 30, (1993), pp 2779.

3. Williams, T.O. "Efficiency and Accuracy Considerations in a Unified Plate Theory with Delaminations," Composite. Structures, 52, (2001) pp. 27-40.

4. Tippetts, T., I.J. Beyerlein, and T.O.Williams "A Multiscale/Cohesive Zone Model for Composite Laminate Impact Damage", American Society Of Composites $17^{\text {th }}$ Technical Conference, Purdue University, IN, Oct. 2002.

5. Park, G., Sohn, H., Farrar, C.R., and Inman, D.J. 2003. "Overview of Piezoelectric impedancebased health monitoring and Path Forward," The Shock and Vibration Digest, accepted for publication.

6. Wait, J.R., Park, G., Sohn, H., Farrar, C.R. "Active Sensing System Development for Damage Prognosis," Proceedings of $4^{\text {th }}$ International Workshop on Structural Health Monitoring, September 15-17, 2003, Stanford, CA, to appear.

7. Todd, M.D., G.A. Johnson, and B.L. Althouse, "A Novel Bragg Grating Sensor Interrogation System Utilizing a Scanning Filter, a Mach-Zehnder Interferometer, and a 3x3 Coupler," Measurement Science and Technology, 12 (7), (2001), pp. 771-777.

8. Johnson, G.A., et al., "Surface effect ship vibro-impact monitoring with distributed arrays of fiber bragg gratings," Proc. of the $18^{\text {th }}$ International Modal Analysis Conf., San Antonio, TX, Feb. 2000.

9. Staszewski, W.J.; K. Worden, K.; R. Wardle, and G.R. Tomlinson, "Fail-safe sensor distributions for impact detection in composite materials" Smart Materials and Structures; 9 (3) (2000), pp.298-303.

10. Barnett, V. and T. Lewis, Outliers in Statistical Data, John Wiley \& Sons, Chichester, 1994.

11. Bishop, C.M., Neural Networks for Pattern Recognition, Oxford University Press, Oxford, UK, 1995.

12. Kramer, M. "Nonlinear Principal Component Analysis Using Autoassociative Neural Networks," AIChE Journal, 37, (1991) pp. 233-243.

13. Fukunaga, K., Statistical Pattern Recognition, Academic Press, San Diego, CA, 1990.

14. Back, A.D. and A.S. Weigend, "A First Application of Independent Component Analysis to extracting Structure from Stock Returns," Int. J. on Neural Systems, 8 (4), (1998), pp. 473-484, 1998.

15. Robertson, A.N., C.R. Farrar and H. Sohn " Singularity Detection for Structural Health Monitoring Using Holder Exponents," accepted for publication in Mechanical Systems and Signal Processing.

16. Sohn, H. C.R. Farrar, and K. Worden, "Novelty Detection Under Changing Environmental Conditions" Proceedings of SPIE's $8^{\text {th }}$ Annual International Symposium on Smart Structures and Materials, Newport Beach, CA, 2001.

17. Sohn, H. C.R. Farrar, N.F. Hunter, and K. Worden, "Structural Health Monitoring Using Statistical Pattern Recognition Techniques" submitted for publication in ASME Journal of Dynamic Systems, Measurement and Control: Special Issue on Identification of Mechanical Systems, 2001.

18. Petsounis, K.A. and S.D. Fassois, " Non-Stationary Functional Series TARMA Vibration Modelling and Analysis in a Planar Manipulator," Journal of Sound and Vibration, 231(5), (2000) pp. 1355-1376.

19. Adak, S. "Time Dependent Spectral Analysis of Non-stationary Time Series," Journal of the American Statistical Association, 93, (1998) pp. 1488-1501.

20. Miller, R.G. Beyond ANOVA: Basics of Applied Statistics, Chapman\&Hall/CRC, New York, 1997.

21. Worden, K., G. Manson, and N.R.J. Fieller, "Damage Detection Using Outlier Analysis," Journal of Sound and Vibration, 229(3), (2000) pp. 647-667. 
22. Humenik, K. and K.C. Gross, "Sequential Probability Ratio Tests for Reactor Signal Validation and Sensor Surveillance Applications," Nuclear Science and Engineering, 105, (1990) pp. 383390.

23. Montgomery, D.C. Introduction to Statistical Quality Control, John Wiley \& Sons, Inc., New York, 1996.

24. Duda, R. O. and P. E. Hart, Pattern Classification and Scene Analysis, John Wiley \& Sons, Inc., New York, 1973.

25. Langley, R.S. "The Dynamic Analysis of Uncertain Structures," Proc. 7th International Conference on Recent Advances in Structural Dynamics, University of Southampton, Southampton, UK, 2000.

26. Egan, J.P. "Signal Detection Theory and ROC Analysis" Academic Press, New York, 1975.

27. Worden, K., D.W. Allen, H. Sohn, and C.F. Farrar, "Extreme Value Statistics for Damage Detection in Mechanical Structures", Los Alamos National Laboratory Report, LA-13903-MS, August 2002.

28. Ljung, L. System Identification-Theory for the Use", Prentice Hall, Upper Saddle River, New Jersey, 1999.

29. Box, G.E., G.M. Jenkins, G.C. Reinsel, Time series analysis - forcasting and control, Prentice Hall, NJ, 1994

30. Wu, C.F.J., and Hamada, M., Experiments: Planning, Analysis, and Parameter Design Optimization, John Wiley and Sons, 2000.

31. Klir, G.J., and B. Yuan, Fuzzy Sets and Fuzzy Logic: Theory and Applications, Prentice Hall, 1995.

32. Shafer, G., A Mathematical Theory of Evidence, Princeton University Press, 1976

33. Ben-Haim, Y., Information-Gap Decision Theory: Decisions Under Severe Uncertainty, Series on Decision and Risk, Academic Press, 2001.

34. McKay, M.D., Beckman, R.J., and Conover, W.J., "A Comparison of Three Methods for Selecting Values of Input Variables in the Analysis of Output from a Computer Code", Technometrics, 21 ( 2), (1979), pp. 239-245.

35. Carlin, B.P., and Chib, S., "Bayesian Model Choice via Markov Chain Monte Carlo," Journal of the Royal Statistical Society, Series B, 77, (1995), pp. 473-484.

36. Chipman, H., Hamada, M., and Wu, C.F.J., "A Bayesian Variable Selection Approach for Analysing Designed Experiments with Complex Aliasing," Technometrics, 39, (1997), pp. 372381.

37. Doebling, S.W., Hemez, F.M., and Schultze, J.F., "Validation of the Transient Structural Response of a Threaded Assembly," Proceedings of the $4^{\text {th }}$ AIAA Non-Deterministic Approaches Forum at the Structures, Structural Dynamics and Materials Conference, Denver, CO, Apr. 22-25, 2002.

38. Kerschen, G., Golinval, J.-C., and Hemez, F.M., "Bayesian Model Screening for the Identification of Non-linear Mechanical Structures," to appear in ASME Journal of Vibration and Acoustics, 2003.

39. Doebling, S.W., "Structural Dynamics Model Validation: Pushing the Envelope," Proceedings of the International Conference on Structural Dynamics Modelling: Test, Analysis, Correlation and Validation, Madeira Island, Portugal, June 3-5, 2002.

40. Madsen, H.O., S. Krenk, N.C. Lind, Methods of Structural Safety, Prentice Hall, New Jersey, 1986. 